\title{
Stimulative Effects of Low Intensity He-Ne Laser Irradiation on the Proliferative Potential and Cell-Cycle Progression of Myoblasts in Culture
}

\author{
Cui-Ping Zhang, ${ }^{1}$ Shao-Dan Li, ${ }^{2}$ Yan Chen, ${ }^{3}$ Yan-Ming Jiang, \\ Peng Chen, ${ }^{5}$ Chang-Zhen Wang, ${ }^{5}$ Xiao-Bing Fu, ${ }^{1}$ Hong-Xiang Kang, \\ Ben-Jian Shen, ${ }^{5}$ and Jie Liang ${ }^{5}$ \\ ${ }^{1}$ Department of Wound Healing and Cell Biology, Burns Institute, The First Affiliated Hospital, \\ General Hospital of Chinese People's Liberation Army, Beijing 100048, China \\ ${ }^{2}$ Institute of Traditional Chinese Medicine, General Hospital of Chinese People's Liberation Army, Beijing 100853, China \\ ${ }^{3}$ Department of Pharmacy, General Hospital of Beijing Military Region, Beijing 100700, China \\ ${ }^{4}$ Department of Ophthalmology, Second Artillery Gerenal Hospital of Chinese People’s Liberation Army, Beijing 100088, China \\ ${ }^{5}$ Department of Electromagnetic and Laser Biology, Institute of Radiation Medicine, Academy of Military Medical Sciences, \\ Beijing 100850, China
}

Correspondence should be addressed to Peng Chen; chenbj2002@sina.com

Received 17 April 2014; Accepted 10 June 2014; Published 21 July 2014

Academic Editor: Timon Cheng-Yi Liu

Copyright (c) 2014 Cui-Ping Zhang et al. This is an open access article distributed under the Creative Commons Attribution License, which permits unrestricted use, distribution, and reproduction in any medium, provided the original work is properly cited.

Low intensity laser irradiation (LILI) was found to promote the regeneration of skeletal muscle in vivo but the cellular mechanisms are not fully understood. Myoblasts, normally quiescent and inactivated in adult skeletal muscle, are a type of myogenic progenitor cells and considered as the major candidates responsible for muscle regeneration. The aim of the present study was to study the effect of LILI on the growth potential and cell-cycle progression of the cultured myoblasts. Primary myoblasts isolated from rat hind legs were cultured in nutrient-deficient medium for 36 hours and then irradiated by helium-neon laser at a certain energy density. Immunohistochemical and flow cytometric analysis revealed that laser irradiation could increase the expression of cellular proliferation marker and the amount of cell subpopulations in the proliferative phase as compared with the nonirradiated control group. Meanwhile, the expressions of cell-cycle regulatory proteins in the laser-treated myoblasts were markedly upregulated as compared to the unirradiated cells, indicating that LILI could promote the reentry of quiescent myoblasts into the cell division cycle. These results suggest that LILI at certain fluences could promote their proliferation, thus contributing to the skeletal muscle regeneration following trauma and myopathic diseases.

\section{Introduction}

Lasers have been widely used in biological and medical fields for many years $[1,2]$. Low intensity laser irradiation (LILI) is usually a laser with milliwatt-grade output power which could produce special biomodulation effects for disease treatment yet without irreversible damage to tissues [3-5]. LILI has achieved positive effects in clinical treatment of wound healing, chronic pain relief, fracture rehabilitation, and so on [6-8]. Studies have demonstrated that LILI could significantly enhance the regeneration process of skeletal muscle in mammals and amphibians $[9,10]$. However, the possible cellular and molecular mechanisms still remain unclear as yet.

In the sight of traditional histopathology, mature skeletal muscle contains postmitotic muscle fibers where they themselves have very limited remodeling capability [9]. Under normal circumstances, adult mammalian skeletal muscle is a stable tissue with very little turnover of nuclei. However, upon injury and with external stimulus, skeletal muscle has the remarkable ability to initiate a repair process involving the activation of various cellular responses. This remodeling process is largely accomplished by myogenic precursors, also 
termed myoblasts, which lie beneath the basement membrane of the muscle fiber and could replicate themselves in growing postnatal muscle where they add myonuclei to enlarge muscle fibers [11, 12]. Yet, in adult skeletal muscle, the number of myoblasts was few and most of them were in quiescent and inactive state, which made tissue regeneration and function repair very difficult following muscle injury. Researchers have found that, under the action of certain growth factors, myoblasts in wounded adult skeletal muscle could be activated to enter the mitotic cycle and proliferate and then differentiate to form new myofibers, thus contributing to the maintenance and restoration of muscle integrity and mass [13].

Previously, it was shown that, relative to nonirradiated control, LILI of the injured site markedly accelerated tissue repair and regeneration of skeletal muscle $[9,10]$. Although the LILI-promoted muscle regeneration has been amply studied in vivo, questions remain, especially regarding the direct effect of this treatment on myoblasts in vitro and the biomodulatory mechanisms by which dormant myoblasts following LILI are activated to participate in muscle regeneration. Therefore, in this experiment, we used myoblasts cultured in vitro to explore the effect of LILI on the proliferative potential and cell-cycle progression of myoblasts and further find out the involved cell-cycle regulatory proteins in this process, with an attempt to provide evidence for the applications of LILI in clinical practice.

\section{Materials and Methods}

2.1. Cell Cultures and Identification. Primary myoblasts were derived from hindlimb muscles of neonatal Wistar rats and cultured in Ham's F-10 nutrient mixture supplemented with $20 \%$ fetal bovine serum (FBS) and incubated in a $\mathrm{CO}_{2}$ enriched (5\%) humid atmosphere at $37^{\circ} \mathrm{C}$. During the first several passages of the primary cultures, myoblasts were enriched by preplating method. Immunocytochemical identification of myoblasts was performed by using a monoclonal antibody to desmin, the muscle-specific intermediate filament protein, and screened under the laser scanning confocal microscope (LSCM).

2.2. Laser Irradiation Protocol. In this experiment, a He$\mathrm{Ne}$ laser with a wavelength of $632.8 \mathrm{~nm}$ was used. Prior to laser irradiation, myoblasts were initially seeded on the cellculture plates at a density of $4 \times 10^{4}$ cells $/ \mathrm{mL}$. At the desired time points, irradiation was performed in the dark at room temperature. Laser irradiation was delivered to the culture plate from above via combination of optical lens. The distance between cells sample and lens was adjusted to make the laser beam diameter the same as the width of culture plates. The power density of irradiation on the cells was measured to be $6 \mathrm{~mW} / \mathrm{cm}^{2}$ and the irradiation time was set to $3 \mathrm{~min}$ based on our preexperiment. The nonirradiated control cells were subjected to the same experimental conditions as the irradiated cells, except for the irradiation.
2.3. Cell Preconditioning and Grouping. To simulate the initial physiological state of myoblasts in vivo and rule out the possible effects of unknown factors in serum, myoblasts were pretreated with serum starvation, so that we can analyze the effect of laser irradiation on myoblasts more accurately. In brief, myoblasts after passage were firstly cultured overnight in growth medium containing 20\% FBS and then switched into serum-free culture medium for 36 hours. The cells which were rendered quiescent and synchronized in G0/G1 phase by serum starvation were then either refed with $10 \%$ FBS (hereafter called "10\% FBS group," the same as below) or irradiated as described above (laser group). The cells neither serum-refed nor irradiated were set as negative control (control group). After serum starvation, myoblasts in laser and control group continued to be kept in the serum-free medium. Meanwhile the cells cultured throughout in serum with 20\% FBS without any treatment (neither starvation nor irradiation) were used as a positive control (20\% FBS group) in this study.

2.4. Flow Cytometric Assay. Myoblasts were harvested $24 \mathrm{~h}$ after laser irradiation or serum refeeding. Following fixation with $75 \%$ ethanol, the cells were digested with DNasefree RNase in phosphate-buffered saline (PBS) containing $5 \mu \mathrm{g} / \mathrm{mL}$ propidium iodide for DNA staining. The propidium iodide fluorescence and forward light scattering were detected by a flow cytometer equipped with CELLQuest software. The percentage of cells in G0/G1 and S/G2/M phases of the cell cycle was calculated, respectively.

2.5. Immunohistochemistry. Myoblasts were fixed with $4 \%$ paraformaldehyde and then incubated with mouse $\mathrm{mAb}$ to proliferate cell nuclear antigen (PCNA) at $4^{\circ} \mathrm{C}$ for 20 hours. After thorough rinsing with PBS, cells were incubated with biotinylated goat anti-mouse IgG and HRP-conjugated streptavidin successively. The peroxidase reaction was performed using AEC as chromogen and examined under a microscope. Three independent experiments in each condition were performed and a total of at least 100 cells were counted for each specimen.

2.6. Western Blotting. After irradiation/refeeding, cells were harvested quickly at desired time points, and the total protein was extracted with lysis buffer. Extracts were quantified with a protein assay kit, fractionated by $6 \%$ SDS/PAGE, and then transferred to a poly membrane. After blocking, membranes were incubated with mouse $\mathrm{mAb}$ to cyclin $\mathrm{A}$ and cyclin $\mathrm{D}$, respectively, and then treated with goat anti-mouse IgG conjugated with horseradish peroxidase. Immune complexes on the membrane were visualized by using diaminobenzidine system. The relative gray values of corresponding bands and $\beta$-actin were compared and semiquantified by image analysis software.

2.7. Statistical Analysis. All of the experimental data were from experiments that were repeated at least three times, unless otherwise indicated. Statistical analysis was performed 


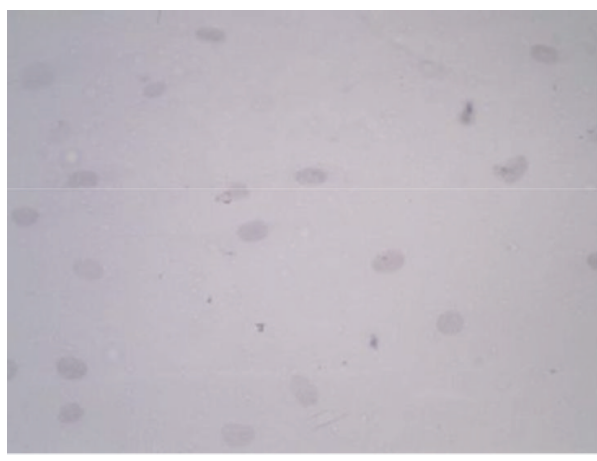

(a)

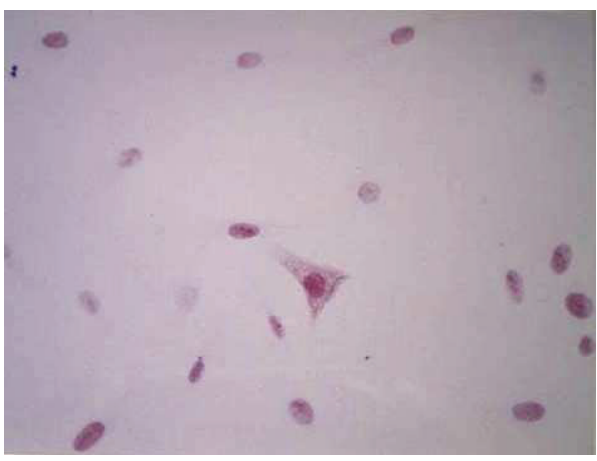

(c)

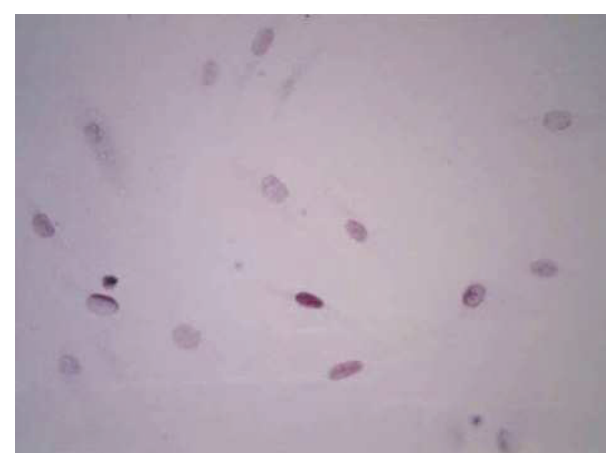

(b)

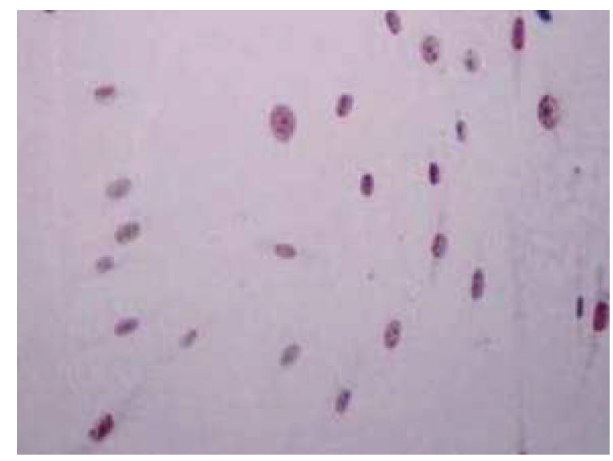

(d)

FIGURE 1: Immunohistochemical detection of PCNA expression in myoblasts after preconditioning with serum starvation (IHC $\times 400)$. (a), (b), (c), and (d) were from control, laser, 10\% FBS, and 20\% FBS group, respectively, the grouping as described above. There was obvious difference in the levels of PCNA expression between each group.

by $t$-test. A statistical probability of $P<0.05$ was considered to be significant.

\section{Results}

3.1. Growth Characteristics of Myoblasts. The enriched primary myoblasts displayed remarkable proliferation potential. Pure myoblast cultures have been expanded beyond $30 \mathrm{popu}-$ lation doublings. There were no detectable changes of growth rate or cell morphology even after extensive proliferation in vitro. Because of the extraordinary growth potential of primary myoblasts under these culture conditions, the serumstarved pretreatment described above was made possible and was not limited by the number of available cells. The cells used for laser irradiation were passaged 3-6 times unless otherwise indicated.

3.2. Effect of LILI on PCNA Expression of Myoblasts. PCNA, proliferating cell nuclear antigen, is the necessary component for DNA duplication of cell chromatosome. Its synthesis and expression were related to cell proliferative cycle. Quantification of PCNA expression is one kind of simple and feasible method for evaluating cell proliferative activity. In this study, the expression of PCNA was detected by immunohistochemical method (IHC) and assessed using semiquantitative analysis. Ours results showed that, 24 hours after laser irradiation, PCNA expression in the laser group was significantly higher than in the control group and the same in the $10 \%$ FBS group but less than that of $20 \%$ FBS group (Figure 1).

3.3. Effect of LILI on Proliferation Index of Myoblasts. Proliferation index (PI) is defined as the total number of cells in all the phases of the cell cycle (G0, G1, S, G2, and M phase) divided by the number of cells that went into division (S, G2, and M phase); that is, $\mathrm{PI}=(\mathrm{S}+\mathrm{G} 2 / \mathrm{M}) /(\mathrm{G} 0 / \mathrm{G} 1+\mathrm{S}$ $+\mathrm{G} 2 / \mathrm{M})$. The proliferation index reflects the percentage of proliferative cells in the $S$ phase and G2/M phase of the cell cycle and can be used with the PCNA expression assay to give a more complete understanding of growth characteristics of myoblasts. As shown in Figures 2 and 3, twenty-four hours after laser irradiation or serum-refeeding, more myoblasts (about 42\%) were in S phase and G2/M phase of the cell cycle as compared with myoblasts in the control group (less than $12 \%$ ), whereas the values were slightly lower than that of myoblasts in $20 \%$ FBS group (approximately $65 \%$ ). The results here suggested that more myoblasts entered the proliferative phase after LILI, which was consistent with the above data.

3.4. Effect of LILI on Expression of Cell-Cycle Regulatory Proteins of Myoblasts. The division cycle of eukaryotic cells is regulated by a family of cell-cycle regulatory proteins (also known as cyclin) and the cyclin-dependent kinases (CDK) complex, of which cyclin D and cyclin A are the key molecules that can trigger cell-cycle entry, thus driving the cells from 


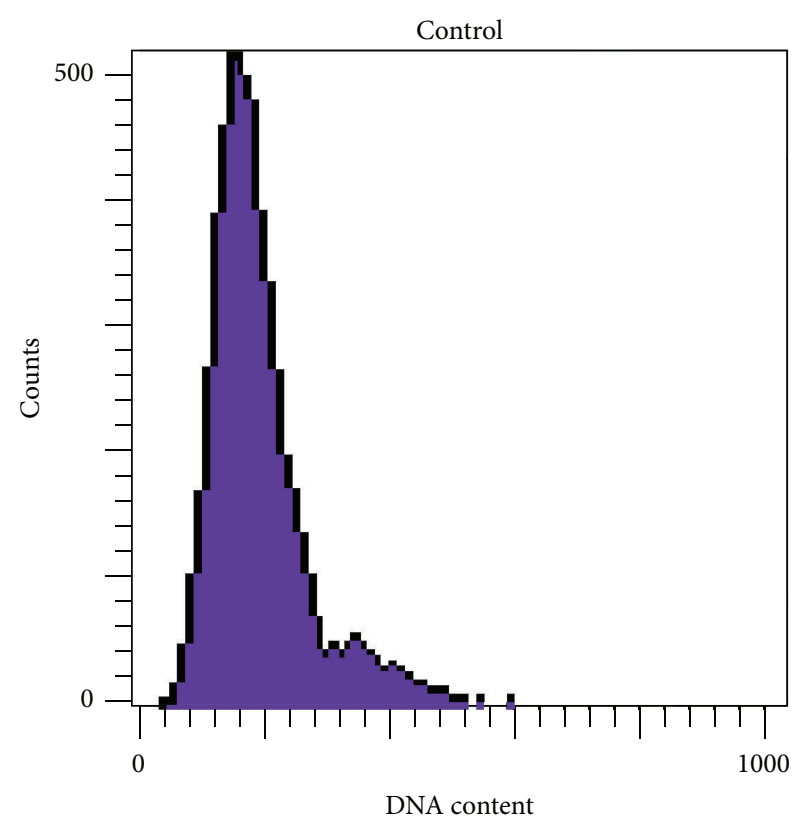

(a)

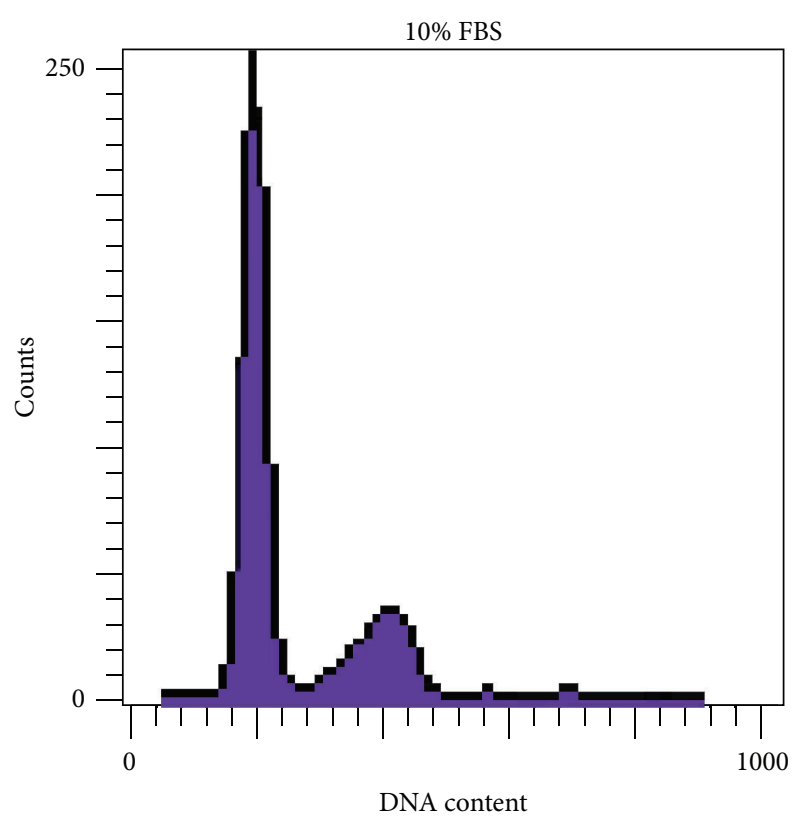

(c)

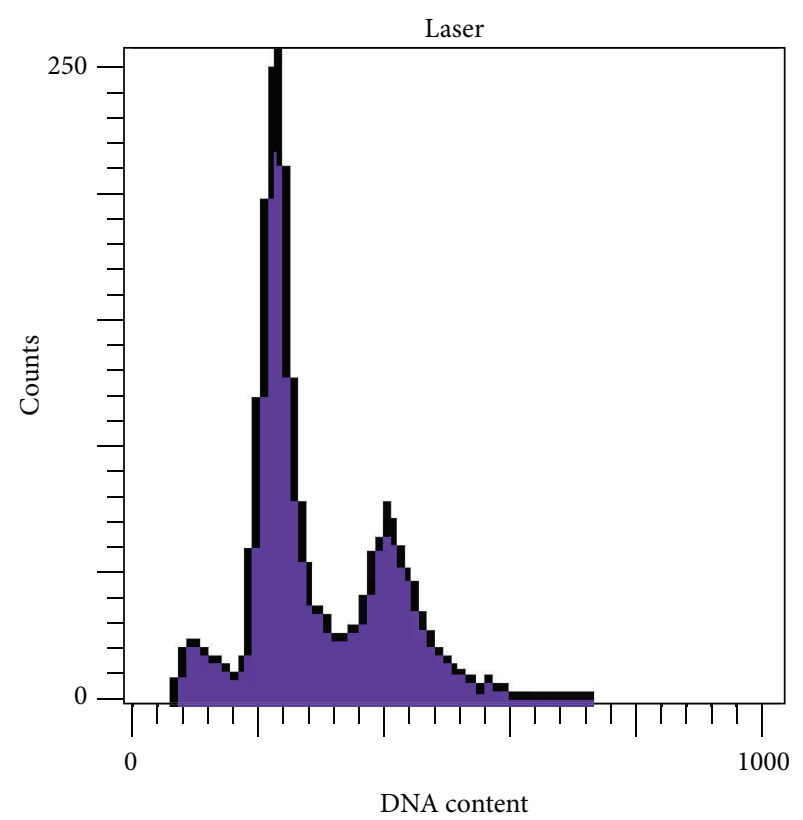

(b)

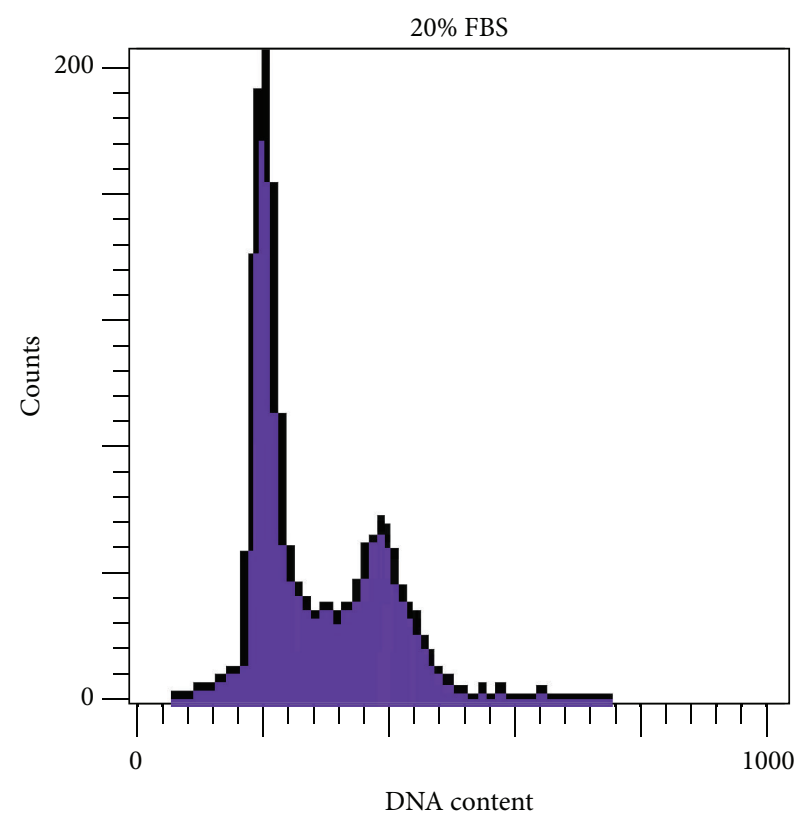

(d)

FIGURE 2: Effects of LLLI on the proliferation index of myoblasts $24 \mathrm{~h}$ after irradiation/refeeding. Typical flow cytometric DNA histograms of the myoblasts from control (a), laser irradiation (b), 10\% FBS (c), and 20\% FBS (d) groups, respectively.

quiescence to the mitotic cycle. The periodic change of their expression is synchronized to cell-cycle progression. As presented in Figure 4, only a slight expression of cyclin D was detected in control cells, whereas the cyclin $\mathrm{D}$ protein levels showed an approximately fivefold or sevenfold increase just $30 \mathrm{~min}$ after irradiation/refeeding in irradiated or serumrefed cells, respectively. The expression of Cyclin A was also induced $2 \mathrm{~h}$ after irradiation in the irradiated cells and the expression level was compatible with its induction in the serum-refed cells, whereas its expression was almost undetectable in nonirradiated control cells. These results suggested that LILI could modulate early cell-cycle regulatory genes and promote cell-cycle entrance from the G0/G1 phase in myoblasts, thereby facilitating the cell-cycle progression and increasing their proliferative potential after serum-starved pretreatment.

\section{Discussion}

It is well known that skeletal muscle repair is a highly synchronized process involving the activation of various cellular responses [11, 14]. Among these, myogenic stem cells, 


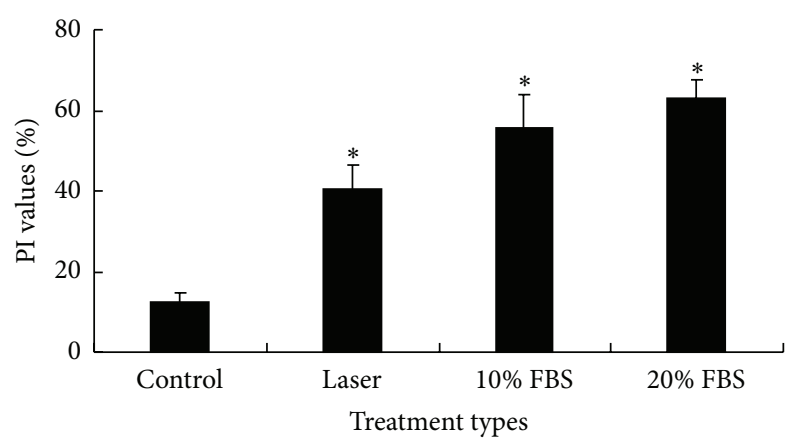

FIGURE 3: Cartogram of flow cytometric analysis about the proliferation index of myoblasts from each group as described above. Data are means \pm SEM. ${ }^{*} P<0.05$, as compared with control.

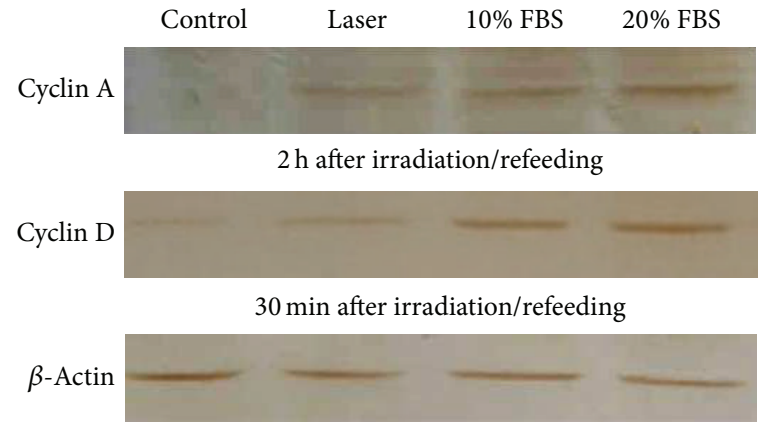

(a)

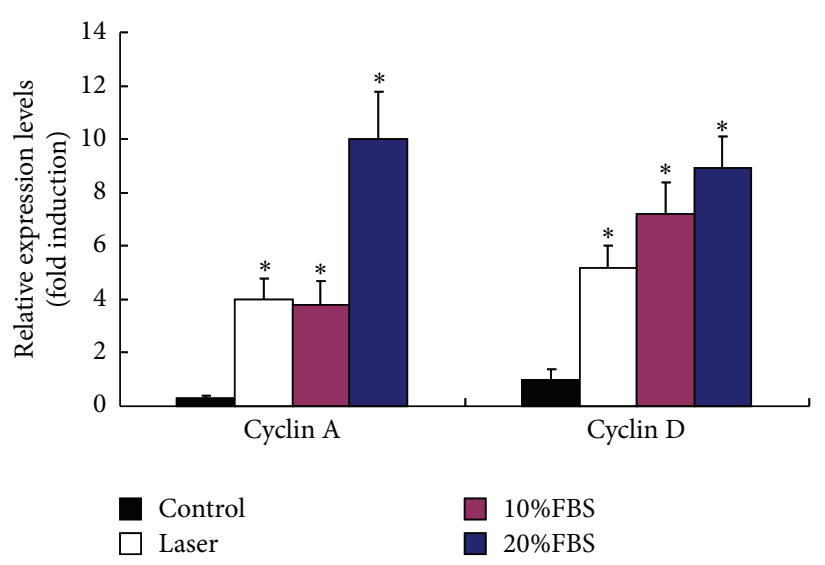

(b)

FIGURE 4: Western blot analysis of cyclin D and cyclin A protein expression after irradiation/refeeding (a). The expression levels of cyclin D and cyclin A were subsequently measured by densitometric analysis (b). The relative protein expression values were normalized to that of $\beta$-actin. ${ }^{*} P<0.05$ compared with nontreated controls.

also known as myoblasts, play a crucial role in this process. In adult skeletal muscle, the number of myoblasts was few and most of them were normally dormant, which made tissue regeneration and function repair difficult following muscle injury [15]. Yet, under the action of exogenous stimulus such as growth factors, these cells could be activated to enter the mitotic cycle and proliferate and further fuse each other to form new muscle fibers, which eventually led to the replacement of injured ones and rapid muscle regeneration [16]. Previous results have demonstrated that following LILI the irradiated site of the injured skeletal muscle featured more newborn myofiber formation, suggesting that the major candidates for muscle regeneration responding to laser irradiation may also be myoblasts $[9,17]$.

So far, however, many laboratory results remain controversial regarding the laser-induced photobiomodulatory effects, and differences of the irradiation parameters and growth conditions used in various studies complicate the issue of making meaningful comparisons [18-20]. In this study, the low-energy helium-neon laser $(632.8 \mathrm{~nm})$ with a power density of approximately $6 \mathrm{~mW} / \mathrm{cm}^{2}$ was used for myoblasts irradiation in vitro. This treatment dosage (power density and irradiation time) was found to be optimal for biostimulation of myoblasts proliferation, based on a series of preliminary experiments with the cell cultures. In our preexperiment, three different energy densities $\left(6 \mathrm{~mW} / \mathrm{cm}^{2}\right.$ with $1 \mathrm{~min}, 3 \mathrm{~min}$ or $10 \mathrm{~min}$ comprising $0.36,1.08$, or $3.6 \mathrm{~J} / \mathrm{cm}^{2}$, resp.) were used. We initially investigated the effect of HeNe laser with different irradiation times on the cell number and myotube formation to determine the optimal energy density for LILI-induced biological effect. We found that irradiation for 3 min could produce maximal biomodulation on myoblasts. Thus, the energy density which gave the best preliminary results $(3 \mathrm{~min})$ was used in the present study where cells were serum starved, given that the cellular response to LILI mainly depended on the initial physiological state of the target cells $[3,4,21,22]$. So in the next experiment, previous to laser irradiation, myoblasts were initially pretreated for $36 \mathrm{~h}$ with serum-deficient medium when grown to nearly confluent and then either refed with $10 \%$ FBS or subjected to laser irradiation. The cells had been previously reported to enter quiescence upon serum starvation which can only maintain survival of young postmitotic cells, so that the physiological state of myoblasts in vivo was simulated 
and the cellular response to LILI could be screened out independently.

However, in this paper, an interesting aspect of the nonirradiated control myoblasts was that these cells, which were maintained throughout in serum-free cultures conditions, also display a little of cell proliferation as shown in Figure 3. In general, the cell cultured in the absence of serum could neither proliferate as usual [23] nor be activated by other stimuli such as LILI [24]. Nevertheless, the growth mediums (Ham's F-10) used in this experiment for myoblasts in vitro culture somehow contained the component of serum according to literature [25], which thus contributed to the low degree of proliferation index of myoblasts in the control group, despite the fact that this kind of cell proliferation in serum-free medium was actually a type of dysfunctional proliferation. Yet, as indicated in our results, this type of dysfunctional proliferation or proliferation imbalance in myoblasts under serum-deficient conditions could be improved notably by LILI. As previously mentioned, in adult skeletal muscle, myoblasts were normally dormant, but once injured they could be activated to initiate the proliferation cycle. If the activated myoblasts could proliferate in a normal way, the injured muscle could automatically renew themselves and LILI can just accelerate this regeneration process by indirect photobiomodulation (PBM) $[4,21]$. Whereas, if these cells were in a state of dysfunctional proliferation (just like under serum-starved conditions in vitro), LILI would directly promote the proliferation of myoblasts in vivo and thus substantially facilitate the recovery of muscle injuries.

Researches have shown that the expression of PCNA, an early cell-cycle protein, which is upregulated in the late G1 phase, could be markedly affected by low intensity laser irradiation [26]. In the present experiment, after $36 \mathrm{~h}$ of serum starvation followed by LILI, PCNA protein levels as well as PI value were also evaluated in primary myoblasts. It has been demonstrated that a growing cell population is composed of cells in all the phases of cell cycle (G1, S, G2, and $\mathrm{M}$ phase), and the estimation of cell subpopulations in $S$ phase and G2/M phase of the cell cycle can be used for cell proliferation evaluation [27]. Normally, there was no obvious PCNA expression in cells of G0/G1 phase, whereas its expression in late G1 phase was substantially increased. The expression reached a peak in $S$ phase and decreased significantly in $\mathrm{G} 2 / \mathrm{M}$ phase. The periodical variations in PCNA expression, as well as PI value, were consistent with the phases of cellular DNA synthesis and thus can reflect proliferation activity of the cultured myoblasts [28]. Our results showed that PCNA expression and the proportion of myoblasts in the S/G2/M phase of cell cycle greatly increased 24 hours after laser irradiation, suggesting that LILI could drive more myoblasts from the quiescent G0/G1 phase entering into mitotic phase and thus enhance their proliferation capacity, which was further confirmed by the upregulated expressions of cyclin D and cyclin A.

As is well known, cyclin D and cyclin A are the initiating factors for G0/G1 conversion and their inducible expression contributed to cell-cycle progression of the LILI-activated myoblasts. Previous studies [26, 29] of mouse myogenic cells under low growth conditions have established that LILI could stimulate cells into the cell cycle up to $24 \mathrm{~h}$ after irradiation and the induction of cyclin D1 expression was detected in the irradiated cells as early as $6 \mathrm{~min}$ after irradiation. Thus, it is more likely that the increased proliferation ability following LILI involves the translation of cyclins that are required for entrance and progression through the Gl phase of the cell cycle. Quantitative results of western blot analysis in this experiment showed that $36 \mathrm{~h}$ after serum withdrawal, cyclin D and cyclin A had only trace expression in control cells, suggesting that myoblasts had been synchronized to the G0/G1 phase after serum starvation; while in a very short time after irradiation, the expressions of cyclin D and cyclin A increased obviously. Thus, these findings along with previous results imply that LILI mediates its stimulative effect on myoblasts proliferation by affecting very early events in the cell cycle, such as the inducible expressions of early cell-cycle regulatory genes.

Further, in a preliminary experiment we also found that LILI with different energy densities had dissimilar influences on myoblasts growth in vitro (data not shown). In this study, LILI for $1 \mathrm{~min}$ had weak promotion on cell number and cell-cycle progression; however, laser irradiation for $10 \mathrm{~min}$ could somehow inhibit myoblasts proliferation, indicating that the biomodulatory effect of LILI on primary myoblasts is dose-dependent, which is also consistent with the biological characteristics of LILI.

In our experiment, the ability of irradiated myoblasts to survive and proliferate in the absence of serum sustenance is remarkable as compared with the nonirradiated cells. Laser irradiation appears to be analogous to the components of serum responsible for myoblasts survival and growth whether myoblasts being dormant in vivo or arrested in G0/G1 phase in vitro and in some ways, it can substitute for the myoblasts serum requirement to reenter the cell cycle from quiescence. It also stands to reason that the cellular response to LILI would be weaken or absent if the myoblasts were initially maintained under serum-sufficient growth conditions instead of the serum-deficient culture medium, particularly considering the fact that, for the laser-induced photobiomodulation, the initial physiological state of the target cells is critical for the treatment response [21, 22, 30, 31].

\section{Conclusion}

Taken together, our studies suggest that myoblasts in culture can be promoted from quiescence into cell proliferation cycle in response to laser irradiation at a certain fluence. These results also indicate that LILI affects early cell-cycle regulatory genes and enhances proliferative potential of myoblasts, thereby increasing cell proliferation and contributing to muscle regeneration in vivo.

\section{Conflict of Interests}

The authors declare that there is no conflict of interests regarding the publication of this paper. 


\section{Authors' Contribution}

Cui-Ping Zhang, Shao-Dan Li, Yan Chen, and Yan-Ming Jiang contributed equally to this work.

\section{Acknowledgments}

This work was supported by the National Natural Science Foundation of China (81171798, 81121004, and 81230041), Beijing Municipal Natural Science Foundation (7142124), and the National Basic Science and Development Program (973 Program, 2012CB518105).

\section{References}

[1] C. Y. Liu and P. Zhu, Intranasal Low Intensity Laser Therapy, People's Military Medical Press, Beijing, China, 2009.

[2] X. Liu, J. Zhang, J. Lu, and T. C. Liu, "Laser acupuncture reduces body fat in obese female undergraduate students," International Journal of Photoenergy, vol. 2012, Article ID 730351, 4 pages, 2012.

[3] T. Karu, The Science of Low-Power Laser Therapy, Gordon and Breach, Amsterdam, The Netherlands, 1998.

[4] T. C. Liu, Y. Y. Liu, E. X. Wei, and F. H. Li, "Photobiomodulation on stress," International Journal of Photoenergy, vol. 2012, Article ID 628649, 11 pages, 2012.

[5] R. Lubart, M. Eichler, R. Lavi, H. Friedman, and A. Shainberg, "Low-energy laser irradiation promotes cellular redox activity," Photomedicine and Laser Surgery, vol. 23, no. 1, pp. 3-9, 2005.

[6] S. B. Rabelo, A. B. Villaverde, R. A. Nicolau, M. A. C. Salgado, M. D. S. Melo, and M. T. T. Pacheco, "Comparison between wound healing in induced diabetic and nondiabetic rats after low-level laser therapy," Photomedicine and Laser Surgery, vol. 24, no. 4, pp. 474-479, 2006.

[7] C. S. Enwemeka, "Intricacies of dose in laser phototherapy for tissue repair and pain relief," Photomedicine and Laser Surgery, vol. 27, no. 3, pp. 387-393, 2009.

[8] S. K. Kazem Shakouri, J. Soleimanpour, Y. Salekzamani, and M. R. Oskuie, "Effect of low-level laser therapy on the fracture healing process," Lasers in Medical Science, vol. 25, no. 1, pp. 7377,2010

[9] J. Nakano, H. Kataoka, J. Sakamoto, T. Origuchi, M. Okita, and T. Yoshimura, "Low-level laser irradiation promotes the recovery of atrophied gastrocnemius skeletal muscle in rats," Experimental Physiology, vol. 94, no. 9, pp. 1005-1015, 2009.

[10] P. C. L. Silveira, L. A. da Silva, C. A. Pinho et al., "Effects of lowlevel laser therapy (GaAs) in an animal model of muscular damage induced by trauma," Lasers in Medical Science, vol. 28, no. 2, pp. 431-436, 2013.

[11] S. B. P. Chargé and M. A. Rudnicki, "Cellular and molecular regulation of muscle regeneration," Physiological Reviews, vol. 84, no. 1, pp. 209-238, 2004.

[12] A. J. Wagers and I. M. Conboy, "Cellular and molecular signatures of muscle regeneration: current concepts and controversies in adult myogenesis," Cell, vol. 122, no. 5, pp. 659-667, 2005.

[13] X. Shi and D. J. Garry, "Muscle stem cells in development, regeneration, and disease," Genes and Development, vol. 20, no. 13, pp. 1692-1708, 2006.
[14] A. Asakura, P. Seale, A. Girgis-Gabardo, and M. A. Rudnicki, "Myogenic specification of side population cells in skeletal muscle," The Journal of Cell Biology, vol. 159, no. 1, pp. 123-134, 2002.

[15] M. Buckingham, "Skeletal muscle formation in vertebrates," Current Opinion in Genetics and Development, vol. 11, no. 4, pp. 440-448, 2001.

[16] M. Buckingham, "Myogenic progenitor cells and skeletal myogenesis in vertebrates," Current Opinion in Genetics and Development, vol. 16, no. 5, pp. 525-532, 2006.

[17] K. Rochlin, S. Yu, S. Roy, and M. K. Baylies, "Myoblast fusion: when it takes more to make one," Developmental Biology, vol. 341, no. 1, pp. 66-83, 2010.

[18] X. G. Liu, Y. J. Zhou, T. C. Liu, and J. Q. Yuan, "Effects of lowlevel laser irradiation on rat skeletal muscle injury after eccentric exercise," Photomedicine and Laser Surgery, vol. 27, no. 6, pp. 863-869, 2009.

[19] A. C. Amaral, N. A. Parizotto, and T. F. Salvini, "Dose-dependency of low-energy HeNe laser effect in regeneration of skeletal muscle in mice," Lasers in Medical Science, vol. 16, no. 1, pp. 4451, 2001.

[20] H. Ma, Y. X. Li, H. L. Chen, Y. X. Cui, and T. C. Y. Liu, "Effects of low-intensity laser irradiation on wound healing in diabetic rats," International Journal of Photoenergy, vol. 2012, Article ID 838496, 7 pages, 2012.

[21] T. C. Y. Liu, D. F. Wu, L. Zhu, P. Peng, L. Liu, and X. B. Yang, "Microenvironment dependent photobiomodulation on function-specific signal transduction pathways," International Journal of Photoenergy, vol. 2014, Article ID 904304, 8 pages, 2014.

[22] T. Karu, "Photobiology of low-power laser effects," Health Physics, vol. 56, no. 5, pp. 691-704, 1989.

[23] M. Wu and T. C. Liu, "Single-cell analysis of protein kinase C activation during anti-apoptosis and apoptosis induced by laser irradiation," Photomedicine and Laser Surgery, vol. 25, no. 2, pp. 129-130, 2007.

[24] M. A. Pogrel, J. W. Chen, and K. Zhang, "Effects of low-ener-gy gallium-aluminum-arsenide laser irradiation on cultured fibroblasts and keratinocytes," Lasers in Surgery and Medicine, vol. 20, no. 4, pp. 426-432, 1997.

[25] D. Loutradis, P. Drakakis, K. Kallianidis et al., "Biological factors in culture media affecting in vitro fertilization, preimplantation embryo development, and implantation," Annals of the New York Academy of Sciences, vol. 900, pp. 325-335, 2000.

[26] N. Ben-Dov, G. Shefer, A. Irinitchev, A. Wernig, U. Oron, and O. Halevy, "Low-energy laser irradiation affects satellite cell proliferation and differentiation in vitro," Biochimica et Biophysica Acta, vol. 1448, no. 3, pp. 372-380, 1999.

[27] L. H. Hartwell and M. B. Kastan, "Cell cycle control and cancer," Science, vol. 266, no. 5192, pp. 1821-1828, 1994.

[28] P. Kurki, M. Vanderlaan, F. Dolbeare, J. Gray, and E. M. Tan, "Expression of proliferating cell nuclear antigen (PCNA)/cyclin during the cell cycle," Experimental Cell Research, vol. 166, no. 1, pp. 209-219, 1986.

[29] G. Shefer, I. Barash, U. Oron, and O. Halevy, "Low-energy laser irradiation enhances de novo protein synthesis via its effects on translation-regulatory proteins in skeletal muscle myoblasts," Biochimica et Biophysica Acta, vol. 1593, no. 2-3, pp. 131-139, 2003. 
[30] Y. Y. Xu, T. C. Y. Liu, and L. Cheng, "Photobiomodulation process," International Journal of Photoenergy, vol. 2012, Article ID 374861, 7 pages, 2012.

[31] T. Karu, L. Pyatibrat, and G. Kalendo, "Irradiation with He-Ne laser increases ATP level in cells cultivated in vitro," Journal of Photochemistry and Photobiology B: Biology, vol. 27, no. 3, pp. 219-223, 1995. 

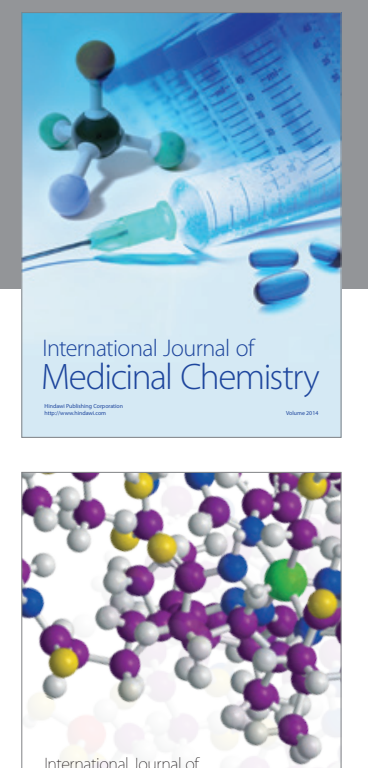

\section{Carbohydrate} Chemistry

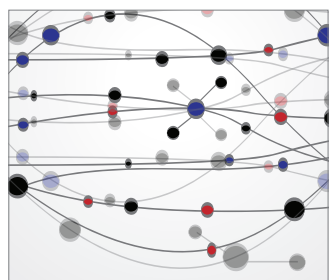

The Scientific World Journal
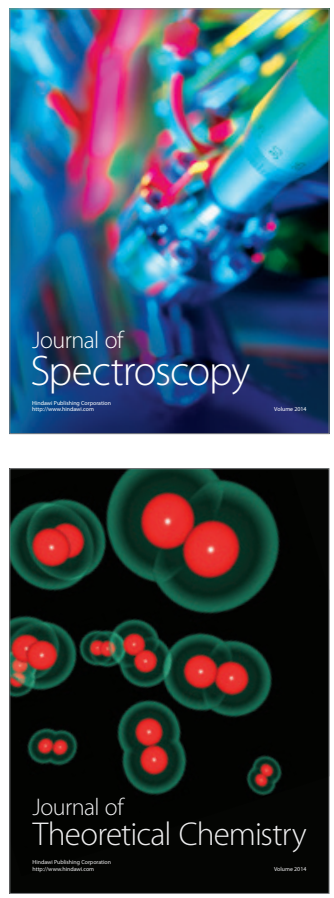
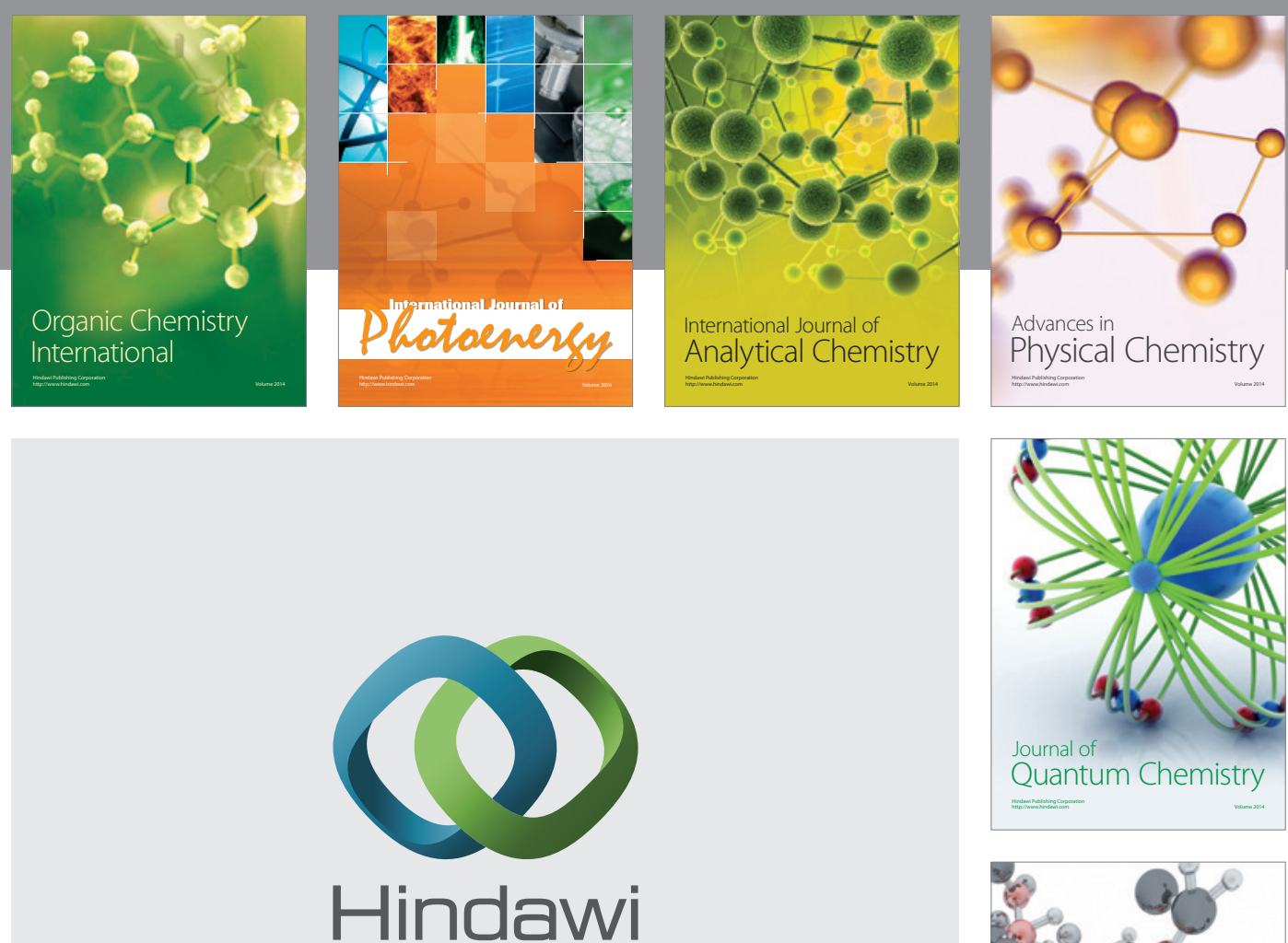

Submit your manuscripts at

http://www.hindawi.com

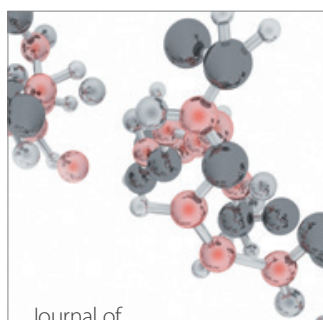

Analytical Methods

in Chemistry

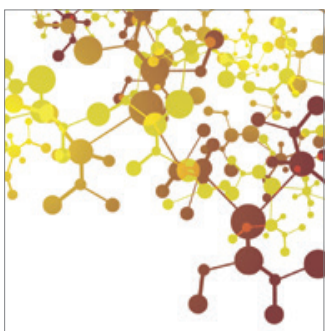

Journal of

Applied Chemistry

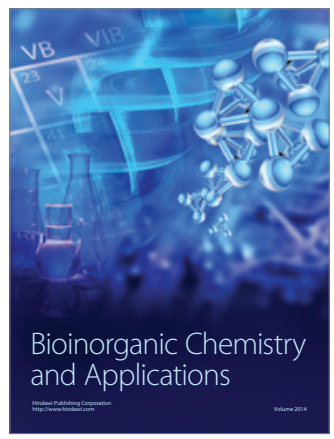

Inorganic Chemistry
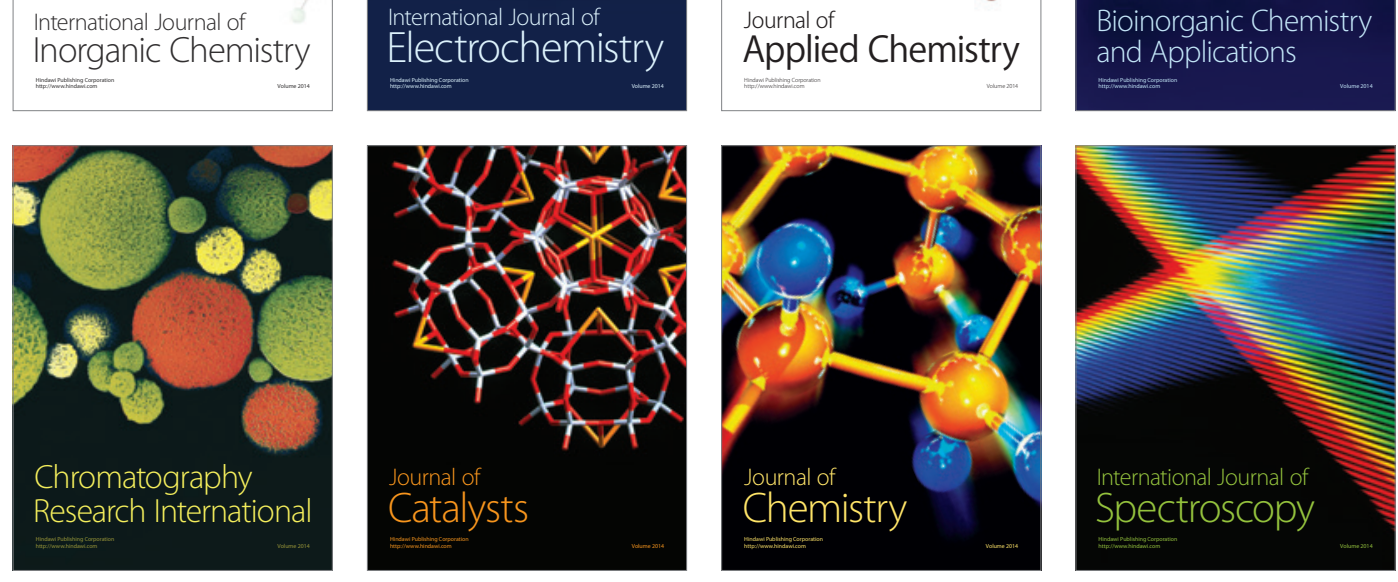